\title{
pxn-1 and pxn-2 May Interact Negatively during Neuronal Development and Aging in C. elegans
}

\author{
Injeong Cho, , Gyu Jin Hwang ${ }^{1,2}$, and Jeong Hoon Cho ${ }^{1, *}$
}

C. elegans has two functional peroxidasins (PXN), PXN-1 and PXN-2. PXN-2 is essential to consolidate the extracellular matrix during development and is suggested to interact with PXN-1 antagonistically. pxn-1 is involved in neuronal development and possibly maintenance; therefore, we investigated the relationship between pxn-1 and pxn-2 in neuronal development and in aging. During neuronal development, defects caused by pxn-1 overexpression were suppressed by overexpression of both pxn-1 and pxn-2. In neuronal aging process, pxn-1 mutants showed less age-related neuronal defects, such as neuronal outgrowth, neuronal wavy processes, and enhanced short-term memory performance. In addition, pxn-2 overexpressing animals retained an intact neuronal morphology when compared with age-matched controls. Consistent with these results, overexpression of both pxn-1 and pxn-2 restored the severe neuronal defects present with pxn-1 overexpression. These results implied that there is a negative relationship between pxn-1 and pxn-2 via pxn-1 regulating pxn-2. Therefore, pxn-1 may function in neuronal development and age-related neuronal maintenance through pxn-2.

\section{INTRODUCTION}

Peroxidasins are members of the peroxidase family with extracellular matrix (ECM) motifs in addition to peroxidase activity. The extracellular motifs contain leucine-rich repeats (LRR) and immunoglobulin-like domains, which are involved in proteinprotein interaction and cell-cell attachment. The first peroxidasin was discovered in Drosophila in 1994 with potential functions proposed in ECM consolidation, phagocytosis, and pathogen defense (Nelson et al., 1994); however, few studies have researched these roles in vivo (Gotenstein et al., 2010; Soudi et al., 2012). Recently, Gotenstein et al reported that peroxidasin PXN-2 is involved in ECM consolidation in Caenorhabditis

\footnotetext{
${ }^{1}$ Department of Biology Education, College of Education, Chosun University, Gwangju 501-759, Korea, ${ }^{2}$ These authors contributed equally to this work.

${ }^{*}$ Correspondence: renocho@chosun.ac.kr

Received 11 May, 2015; revised 4 June, 2015; accepted 8 June, 2015; published online 21 July, 2015
}

Keywords: antagonistic relationship, $C$. elegans, neuronal aging, neuronal development, pxn-1, pxn-2 elegans (C. elegans). PXN-2 is essential for muscle-epidermal attachment during embryogenesis and the maintenance of basement membrane integrity at later stages of development (Gotenstein et al., 2010) by mediating sulfilimine bonds to reinforce the collagen IV network (Fidler et al., 2014). Moreover, this study suggested that PXN-2 could have a negative relationship with another peroxidasin, PXN-1 (Gotenstein et al., 2010).

Our previous study has shown GABAergic neuronal defects in pxn-1 mutants during neuronal development (Lee et al., 2015). Moreover, it has been speculated that pxn-1 was involved in the maintenance of neuronal integrity with age because pxn-1 is expressed throughout the lifespan of $C$. elegans (Lee et al., 2015). There has been controversy regarding neuron deterioration in aging; however, recent studies have shown age-dependent neuronal defects, such as misshapen neuronal soma, extra neurite branching, axon beading, blebbing, bubblelike lesions, and wavy processes in $C$. elegans (Chen et al., 2013; Pan et al., 2011; Tank et al., 2011; Toth et al., 2012). Age-related morphology defects are more prominent in mec-1 and mec-5 mutants, which are involved in nerve attachment, than in other mechanosensory-related mutants (Pan et al., 2011). In addition, the ECM component can stimulate or inhibit neuronal regeneration in the central nervous system (Busch and Silver, 2007); therefore, ECM structure integrity might contribute to both neuronal development and aging. PXN-2 is involved in ECM consolidation during embryogenesis in $C$. elegans and PXN-1 mutants show neuronal defects during neuronal development; therefore, it is likely that peroxidasins play a role in neuronal maintenance and aging through the regulation of ECM integrity. In the present study, we investigated the relationship between pxn-1 and pxn-2 in neuronal development and aging in $C$. elegans.

\section{MATERIALS AND METHODS}

\section{Strains and constructs to observe neuronal defects}

Wild type Bristol N2 strain, pxn-1(ok785) deletion mutant, CZ1200 juls76 (Punc-25::gfp, a GABAergic motor neuron marker) (Jin et al., 1999), and CZ10175 zdls5 (Pmec-4::gfp, a mechanosensory neuron marker) were obtained from the Caenorhabditis Genetics Center (CGC), at the University of Minnesota. C. elegans were cultured according to published protocols (Brenner, 1974).

The $7.2 \mathrm{~kb}$ of $p x n-1$ and $7 \mathrm{~kb}$ of pxn-2 genomic DNAs were amplified by polymerase chain reaction (PCR) and inserted to the Fire vector (pPD95.77). For overexpressing constructs, 
approximately $3 \mathrm{~kb}$ of the $5^{\prime}$-upstream region of pxn-1 and 2.2 $\mathrm{kb}$ upstream of $p x n-2$ were used in this study. The constructs were Ppxn-1::pxn-1::GFP and Ppxn-2::pxn-2::mCherry (gfp of the vector was replaced by $m C h e r r y)$. For ectopic overexpression, a heat shock promoter from the Fire vector (pPD49.83) was used to construct to Phs::pxn-1::GFP, Phs::pxn-2::mCherry. Microinjection was performed as previously described (Mello and Fire, 1995) and the plasmid pRF4 containing a dominant gene (rol-6) was co-injected with the constructs into juls76 (Punc$25:: g f$ ) worms. For ectopic overexpression, the transgenic worms were subjected to heat shock at $30^{\circ} \mathrm{C}$ for $1.5 \mathrm{~h}$ every $12 \mathrm{~h}$.

For RNAi bacterial feeding constructs, the pxn-2 genomic DNA (1.4kb, cut by Bgll and Kpnl) was inserted into the Fire feeding vector (L4440). The RNAi constructs were introduced to HT115(DE3) bacteria. The feeding experiments were performed as described in Kamath et al. (2001).

We scored an individual adult worm as a single neuronal defect when it displayed at least one defect, such as incorrect handedness, breakage, tangling, branching, or defasciculation (Lee et al., 2015). All observations were conducted under a fluorescent microscope (80i-DS-Fi1, Nikon).

\section{Imaging and quantification of neuronal aging}

zdls5 and zdls5;pxn-1 (pxn-1 mutants crossed with zdls5) worms were treated with pxn-2 RNAi bacteria or empty vector (L4440) bacteria. They were cultured and observed for imaging or quantification of aged neurons under the fluorescence microscope.

\section{Short-term associative memory assay}

N2 with gfpRNAi, pxn-1 with gfpRNAi, N2 with pxn-2 RNAi, and pxn-1 with pxn-2 RNAi worms were cultured as described above before the memory assay was conducted. The worms were washed in M9 buffer 3-4 times. After starvation at room temperature for $1 \mathrm{~h}$, worms were transferred to conditioning plates that provided HT115 (DE3) as food and 10\% butanone and incubated for $1 \mathrm{~h}$ at room temperature. The worms were then transferred to a normal growth plate colonized with HT115(DE3) alone and incubated for $1 \mathrm{~h}$. After incubation, the animals were transferred to assay plates that were marked and spotted as origin, $10 \%$ butanone and $95 \%$ ethanol spots according to the protocols (Bargmann et al., 1993; Kauffman et al., $2010 ; 2011)$. After $1 \mathrm{~h}$ of incubation, the worms in each spot were counted. The Chemotaxis Index $(\mathrm{Cl})$ was calculated as $\mathrm{Cl}$ $=\left(\left[\mathrm{n}_{\text {Butanone }}\right]-\left[\mathrm{n}_{\text {EtOH }}\right]\right) /([$ Total - nOrigin $])$.

\section{RESULTS AND DISCUSSION}

\section{pxn-1;pxn-2 double overexpression compensates the} neuronal defects in pxn-1 single overexpression during neuronal development

Overexpression of pxn-1 in worms results in more prominent neuronal defects during development than in pxn-1 mutants (Lee et al., 2015); therefore, we used pxn-1 overexpressing worms with either its own promoter or a heat shock promoter to assess neuronal defects (Figs.1A and 1B). Consistent with our previous study, pxn-1 overexpression (Ppxn-1::pxn-1) resulted in increased axonal defects when compared with control worms (74 and 36\%, respectively; Fig. 1A). pxn-2 overexpressing worms (Ppxn-2::pxn-2) showed slightly increased defects (44\%; Fig. 1A). The overexpression of both pxn-1 and pxn-2 (Ppxn-1::pxn-1+Ppxn-2::pxn-2) attenuated the neuronal defects when compared with pxn-1 overexpression alone (47\%; Fig. $1 \mathrm{~A})$. There was a $45 \%$ recovery of neuronal defects in pxn-
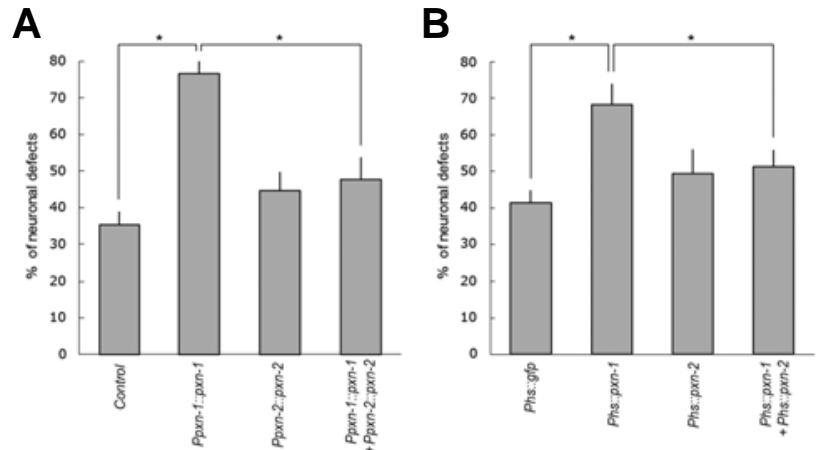

Fig. 1. pxn-1 and pxn-2 double overexpression suppresses the effect of $p x n-1$ overexpression in developing neurons. (A) Neuronal defects during development are presented as the percentage of the total number of neurons counted in overexpressing animals: control (juls76 [Punc-25::gfp]), pxn-1 overexpression (Ppxn-1::pxn-1), pxn2 overexpression (Ppxn-2::pxn-2), and pxn-1 and pxn-2 overexpression (Ppxn-1::pxn-1+Ppxn-2::pxn-2). (B) Neuronal defects during development are presented as a percentage in various ectopic overexpressing worms: Phs::gfp, pxn-1 overexpression (Phs::pxn1), pxn-2 overexpression (Phs::pxn-2), and pxn-1 and pxn-2 overexpression (Phs::pxn-1+Phs::pxn-2). All experiments were performed in juls76 background. $\mathrm{n}>300$ worms per line. Error bar $=$ STD. ${ }^{*} p<0.01$; Student's $t$-test.

1;pxn-2 double overexpressing worms when compared with pxn-1 overexpressing worms. These results were replicated using the ectopic overexpressing animals, Phs::pxn-1, Phs:: pxn-2, and Phs::pxn-1 + Phs::pxn-2 (Fig. 1B). The recovery pattern of double overexpressing animals was similar between ectopic and cis-overexpression, although the rate differed (45\% and $21 \%$ in cis- and ectopic overexpression, respectively). These results suggested that $p x n-1$ has an antagonistic relationship with pxn-2 in neuronal development, which is consistent with the results of Gotenstein et al. (2010).

\section{pxn-1 deletion mutation slows the progression of neuronal aging}

Our previous study suggested that pxn-1 may be involved in neuronal maintenance with age (Lee et al., 2015). In addition, recent studies have demonstrated that dopaminergic, GABAergic motor, and mechanosensory neurons undergo morphological changes during aging, including soma outgrowth, ectopic neurite branching, axon beading, lesioning, blebbing, and wavy process development (Pan et al., 2011; Tank et al., 2011; Toth et al., 2012). Mechanosensory neurons are advantageous because they can be easily observed and are well-studied (Chen et al., 2013); therefore, we chose touch receptor neurons, anterior lateral microtubule cells (ALM), and posterior lateral microtubule cells (PLM) to investigate neuronal aging. The morphology of touch neurons was observed during aging in controls, pxn-1 mutants, pxn-2 RNAi worms, and pxn-1;pxn-2 RNAi worms by using the zdls5(Pmec-4::gfp) background. Control worms (zdls5) presented intact ALMs and PLMs at the adult stage (day 1; Figs. 2A and 2B). Representative images of ALM outgrowth on day 10 and day 15 are shown in Figs. 2C and 2D (arrow). Figures 2C and 2E represent the wavy processes of PLMs on day 10 and day 15 (arrowhead). As shown in Fig. 2, the PLM wavy phenotype was observed more frequently and severely on day 15 than day 10; therefore, this deteriorating 


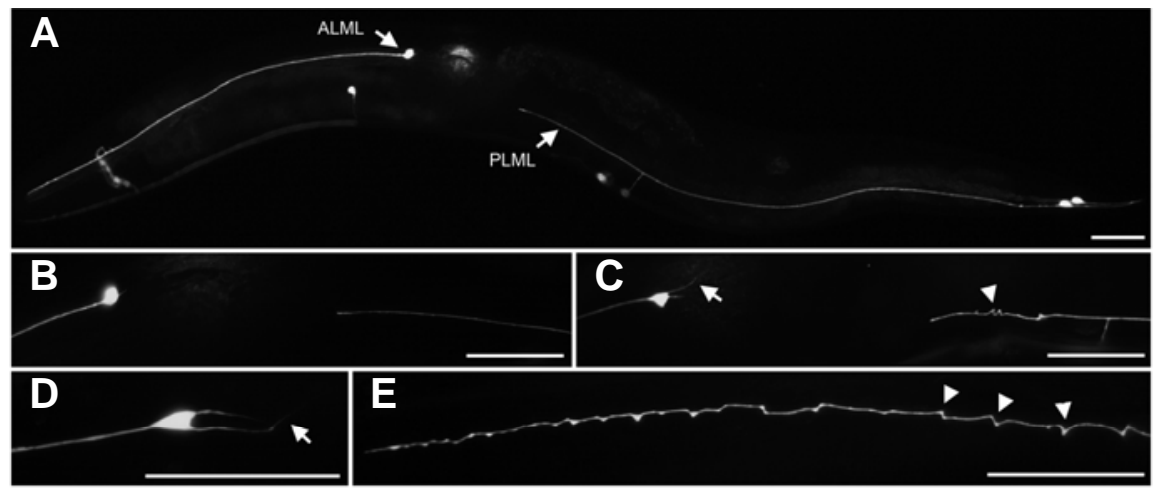

$\boldsymbol{F}$

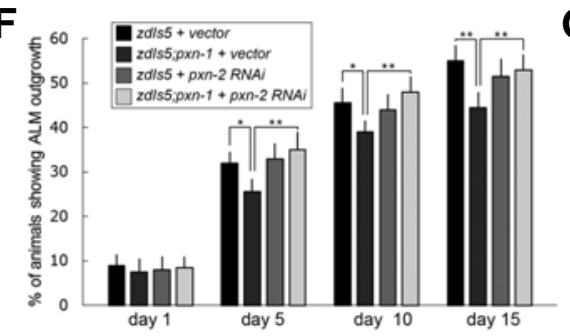

G

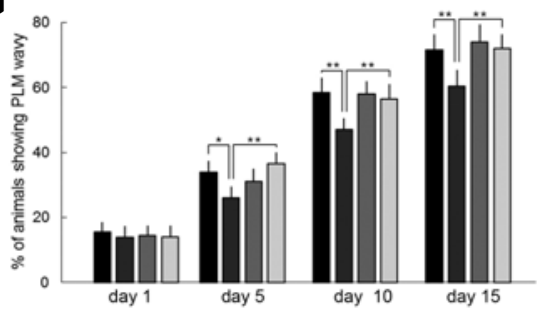

Fig. 2. Neuronal defects increase with age. (A, B) A representative image of a mechanosensory neuron ALM, and PLM in a control worm (vector only in zdls5) on day 1 , young adult stage. (C-E) Representative ALM and PLM on day 10 (C) and on day 15 (D, E, respectively). The arrowheads indicate a wavy phenotype ( $C$ and $E$ ) and the arrows represent an outgrowth $(C, D)$. Scale bar $=50 \mu \mathrm{m}$. $(F, G)$ ALM outgrowth and PLM wavy neurons are presented as a percentage on days $1,5,10$, and 15 in control, pxn-1 mutant (ok785), pxn-2 RNAi treated worms and pxn-1 treated with pxn-2 RNAi worms. All experiments were performed in zdls5(Pmec-4:.gfp) background. $\mathrm{n}>300$ worms per line. Error bar $=$ STD. ${ }^{*} p<0.05 ;{ }^{* *} p<0.01$; Student's $t$-test. phenotype was correlated with aging. To compare neuronal aging among the genotypes, we quantified neuronal defects, such as outgrowth and wavy processes on days 1, 5, 10, and 15 (Figs. 2F and 2G). On day 1, there were no significant differences in neuronal defects in all genotypes (Figs. 2F and 2G); they all contained relatively healthy neurons. As their age progressed, neuronal outgrowth and the frequency of wavy processes increased in all genotypes. In particular, all genotypes showed a sharp increase in ALM outgrowth to $\geq 20 \%$ from days 1 to 5 , then the rate of increase slowed (Fig. $2 F$ ). Considering the short life span of $C$. elegans (2-3 weeks), $53 \%$ neuronal outgrowth by day 15 in control worms was expected. pxn-1 mutants showed consistently lesser neuronal outgrowth throughout the aging process when compared with control worms (Fig. 2F). By contrast, the neuronal outgrowth rate of $p x n-2$ RNAi worms was similar to that of control worms throughout the aging process. Deficient worms with both pxn-1 and pxn-2 (pxn-1;pxn-2) also showed an outgrowth frequency at the control level during aging, further confirming an antagonistic relationship between pxn-1 and pxn-2. There was a similar pattern found with an additional indicator of neuronal aging, PLM wavy processes (Fig. 2G). The increase in wavy processes was continued to day 15 steadily. Consistent with the ALM outgrowth results, pxn-1 mutant animals showed less wavy neurons when compared with control worms. The frequency of wavy neurons in pxn-2 RNAi and pxn-1;pxn-2 RNAi animals was not significantly different from control worms at any time-point (Fig. 2G). These results suggested that pxn-1 mutants showed a slower progression of neuronal aging. Moreover, the restored phenotypes in pxn-1;pxn-2 RNAi worms indicated that pxn-1 and pxn-2 have an antagonistic relationship in neuronal aging.

\section{pxn-2 overexpression reduces age-related neuronal} defects

To confirm the negative relationship between pxn-1 and pxn-2 in neuronal aging, we tested worms overexpressing $p x n-1$ and pxn-2. We used the following genotypes: Ppxn-1::pxn-1::gfp,
Ppxn-2::pxn-2::mCherry, Ppxn-1::pxn-1::gfp + Ppxn-2::pxn-2:: mCherry, and control worms on a zdls5 background with the rol-6 marker. We quantified ALM outgrowth, PLM wavy processes, and PLM branching. There was significantly increased ALM outgrowth on day 15, and PLM wavy processes and branching on days 5 and 15 in pxn-1 overexpressing worms (Figs. 3A-3C). Interestingly, pxn-2 overexpression decreased the ALM defects and PLM wavy processes when compared with control worms on day 5 and day 15 (Figs. 3A and 3B). Furthermore, the frequency of wavy neurons in pxn-2 overexpressing worms on day 15 was similar to that in control worms on day 5.The occurrence of PLM branching in pxn-2 overexpressing worms was not significantly different from control worms on day 5 or 15 (Fig. 3C). In contrast, pxn-1 overexpression showed various PLM defects, including wavy processes, branching, and blebbing (arrows, arrowheads, and open arrow respectively in Fig. 3D) ; therefore, the representative image of PLM defects on day 15 in pxn-1 overexpressing worms is shown in Fig. 3D. Despite low occurrence of PLM branching, pxn-1 overexpression dramatically increased defects regardless of time and the type of defects (Fig. $3 \mathrm{C}$ ). There were no significant differences in ALM or PLM phenotypes in pxn-1;pxn2 double overexpressing worms when compared with controls on day 5 and day 15 (Figs. 3A-3C).

pxn-1 deletion mutation attenuates the age-related decline in short-term memory

According to Kauffman et al. (2010) defects in neuronal morphology can indicate both structural and functional neuronal deterioration. Loss of learning and memory is an early recognizable indicator of neuronal aging, followed by chemotaxis and motility loss (Kauffman et al., 2010). We tested short-term memory performance to assess whether the observed morphological defects of neurons reflected a loss of function. We tested the following genotypes: N2 with gfp RNAi, pxn-1 with gfpRNAi, N2; pxn-2 RNAi, and pxn-1;pxn-2 RNAi on days 1, 3, 5, and 7 (Fig. 4). Short-term memory ability was measured via the 
$\boldsymbol{A}$

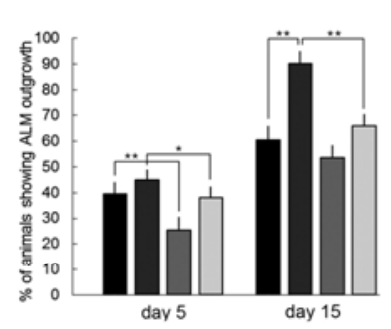

$B$

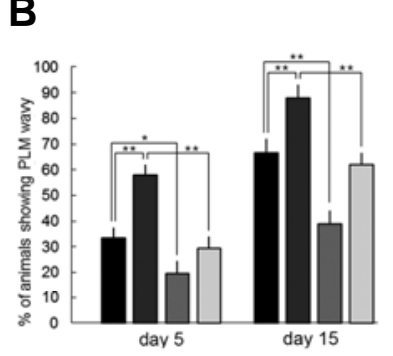

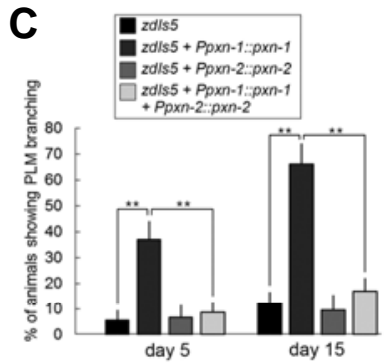
day 15

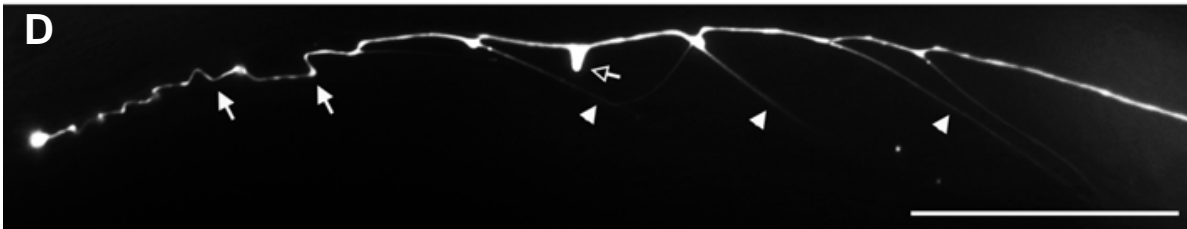

Fig. 3. pxn-1 overexpression exacerbates age-related neuronal defects. (AC) ALM outgrowth, PLM wavy neurons, and PLM branching in each genotype are presented as a percentage on days 5 and 15 respectively in control (zdls5), pxn-1 overexpressing, pxn-2 overexpressing, and $p x n-1$ and $p x n-2$ overexpressing worms. (D) A representative image of a mechanosensory neuron PLM in a pxn-1 overexpressing worm on day 5 . Arrows and arrowheads represent wavy process and branching respectively. Open arrow represents a blebbing. Scale bar $=50 \mu \mathrm{m} . \mathrm{n}>300$ worms per line. Error bar $=$ STD. ${ }^{*} p<$ $0.05 ;{ }^{* \star} p<0.01$; Student's $t$-test.

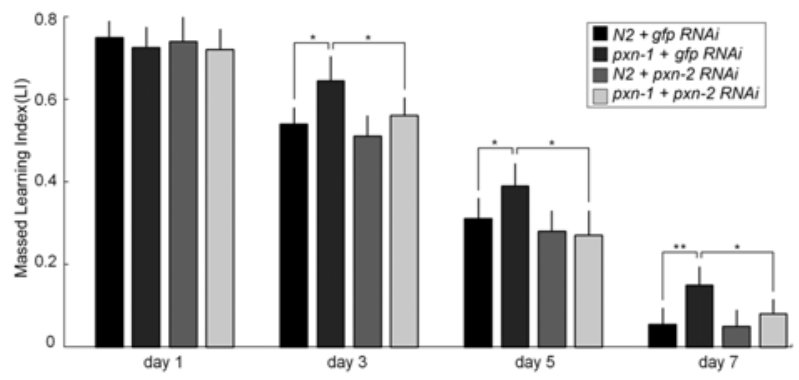

Fig. 4. Age-related short term memory loss is attenuated in pxn-1 mutants. The massed learning index (LI) represents a measurement of short term memory and it is calculated by chemotaxis responses. The measurements are performed on day 1, 3, 5, and 7 in control (N2 with gfp RNAi), pxn-1 mutant, N2 with pxn-2 RNAi treated worms and pxn-1 treated with pxn-2 RNAi worms. $\mathrm{N}>300$ worms per line. Error bar $=$ STD. ${ }^{*} p<0.05 ;{ }^{* *} p<0.01$; Student's $t$ test.

Chemotaxis response and the results were presented as a massed learning index $\left(\mathrm{LI}=\mathrm{Cl}_{\text {Trained }}-\mathrm{Cl}_{\text {Naive }}\right)$, derived from the observed chemotaxis index [Cl,(Kauffman et al., 2011)]. On day 1 , all genotypes showed similar LIs. N2 control and pxn-2 RNAi worms performed slightly better than the other groups, but this was not statistically significant (Fig. 4). N2 control and pxn-2 RNAi worms showed significant decrease in memory function on day 3 and further decrease on day 5 and 7 . However, pxn-1 mutants consistently showed significantly enhanced performance in the short-term memory test when compared with the control worms from day 3 to day 7 . Moreover, pxn-1;pxn-2 RNAi worms showed similar pattern of the N2 decrease in memory function throughout aging, which indicated that the pxn-1;pxn-2 double deficiency suppressed the effects of the pxn-1 mutation. These results were consistent with the ALM and PLM neuronal defect results, which further confirm the antagonistic relationship of $p x n-1$ with $p x n-2$.

The consistent decrease in short-term memory in all genotypes was comparable to the results in Kauffman et al. (2010) suggesting that memory loss is followed by neuronal deterioration (Kauffman et al., 2010). Our results suggested that pxn-2 is important in neuronal development and aging, and pxn-1 negatively regulates $p x n-2$ function. Moreover, the neuronal defects in both double deficiency and double overexpression conditions were recovered to the level of defects in wild type worms; therefore, the relation between $p x n-1$ and $p x n-2$ could be important to peroxidasins' function in neuronal development and in aging. In addition, Peterfi et al. (2014) have shown that the vascular peroxidasin-like protein (PXDNL), a peroxidasin without peroxidase activity, binds vascular peroxidasin (PXDN) to inhibit peroxidase activity in the human heart (Peterfi et al., 2014). This study suggests that PXN-1 and PXN-2 might interact directly or indirectly to regulate peroxidase activity in $C$. elegans. However, it is possible that pxn-1 functions independently or there are other master controller(s) that maintain neuronal integrity during aging.

\section{ACKNOWLEDGMENTS}

We thank the Caenorhabditis Genetics Center (CGC) at the University of Minnesota, USA for the worm strains and Andrew Fire for the Fire vectors. This research was supported by the National Research Foundation of Korea (NRF) grant 20100012373.

\section{REFERENCES}

Bargmann, C.I., Hartwieg, E., and Horvitz, H.R. (1993). Odorantselective genes and neurons mediate olfaction in $C$. elegans. Cell 74, 515-527.

Brenner, S. (1974). The genetics of Caenorhabditis elegans. Genetics 77, 71-94.

Busch, S.A., and Silver, J. (2007). The role of extracellular matrix in CNS regeneration. Curr. Opin. Neurobiol. 17, 120-127.

Chen, C.H., Chen, Y.C., Jiang, H.C., Chen, C.K., and Pan, C.L. (2013). Neuronal aging: learning from C. elegans. J. Mol. Signal. $8,14$.

Fidler, A.L., Vanacore, R.M., Chetyrkin, S.V., Pedchenko, V.K., Bhave, G., Yin, V.P., Stothers, C.L., Rose, K.L., McDonald, W.H., Clark, T.A., et al. (2014). A unique covalent bond in basement membrane is a primordial innovation for tissue evolution. Proc. Natl. Acad. Sci. USA 111, 331-336.

Gotenstein, J.R., Swale, R.E., Fukuda, T., Wu, Z., Giurumescu, C.A., Goncharov, A., Jin, Y., and Chisholm, A.D. (2010). The C. elegans peroxidasin PXN-2 is essential for embryonic morphogenesis and inhibits adult axon regeneration. Development 137, 3603-3613.

Jin, Y., Jorgensen, E., Hartwieg, E., and Horvitz, H.R. (1999). The Caenorhabditis elegans gene unc-25 encodes glutamic acid decarboxylase and is required for synaptic transmission but not synaptic development. J. Neurosci. 19, 539-548.

Kamath, R.S., Martinez-Campos, M., Zipperlen, P., Fraser, A.G., and Ahringer, J. (2001). Effectiveness of specific RNAmediated interference through ingested double-stranded RNA 
in Caenorhabditis elegans. Genome Biol. 2, RESEARCH0002.

Kauffman, A.L., Ashraf, J.M., Corces-Zimmerman, M.R., Landis, J.N., and Murphy, C.T. (2010). Insulin signaling and dietary restriction differentially influence the decline of learning and memory with age. PLoS Biol. 8, e1000372.

Kauffman, A., Parsons, L., Stein, G., Wills, A., Kaletsky, R., and Murphy, C. (2011). C. elegans positive butanone learning, shortterm, and long-term associative memory assays. J. Vis. Exp. 11, pii: 2490,

Lee, J., Bandyopadhyay, J., Lee, J.I., Cho, I., Park, D., and Cho, J.H. (2015). A role for peroxidasin PXN-1 in aspects of $C$. elegans development. Mol. Cells 38, 51-57.

Mello, C., and Fire, A. (1995). DNA transformation. Methods Cell Biol. 48, 451-482.

Nelson, R.E., Fessler, L.I., Takagi, Y., Blumberg, B., Keene, D.R., Olson, P.F., Parker, C.G., and Fessler, J.H. (1994). Peroxidasin: a novel enzyme-matrix protein of Drosophila development. EMBO J. 13, 3438-3447.

Pan, C.L., Peng, C.Y., Chen, C.H., and Mclntire, S. (2011). Genetic analysis of age-dependent defects of the Caenorhabditis elegans touch receptor neurons. Proc. Natl. Acad. Sci. USA 108, 9274-9279.

Peterfi, Z., Toth, Z.E., Kovacs, H.A., Lazar, E., Sum, A., Donko, A., Sirokmany, G., Shah, A.M., and Geiszt, M. (2014). Peroxidasinlike protein: a novel peroxidase homologue in the human heart. Cardiovasc Res. 101, 393-399.

Soudi, M., Zamocky, M., Jakopitsch, C., Furtmuller, P.G., and Obinger, C. (2012). Molecular evolution, structure, and function of peroxidasins. Chem. Biodivers 9, 1776-1793.

Tank, E.M., Rodgers, K.E., and Kenyon, C. (2011). Spontaneous age-related neurite branching in Caenorhabditis elegans. J. Neurosci. 31, 9279-9288.

Toth, M.L., Melentijevic, I., Shah, L., Bhatia, A., Lu, K., Talwar, A., Naji, H., Ibanez-Ventoso, C., Ghose, P., Jevince, A., et al (2012). Neurite sprouting and synapse deterioration in the aging Caenorhabditis elegans nervous system. J. Neurosci. 32, 87788790 . 